\title{
When the Direct Electrical Cortical Stimulation Failed: A Case Report
}

\author{
Mostafa El Khashab ${ }^{\mathrm{a}, \mathrm{f}}$, Wen-Ching Liu ${ }^{\mathrm{b}}$, Arno Fried ${ }^{\mathrm{a}}$, Theresa M. Gabay ${ }^{\mathrm{c}}$, \\ Sean D Pierce ${ }^{\mathrm{d}}$, George A Ghacibeh ${ }^{\mathrm{e}}$
}

\begin{abstract}
Direct electrical cortical stimulation is often considered the "gold standard" to identify motor or other eloquent cortices during surgical procedures. However, this method sometimes fails or is insufficient by itself, and the use of other methods can be very helpful in reducing post-operative morbidity. We report the case of a 28 -yearold patient who was considered for surgical resection of epileptic focus due to intractable seizure. During surgery both direct electrical stimulation and cortical somatosensory evoked potentials failed to identify the motor and sensory areas for unclear reasons. The surgical plan for resection of his seizure focus was aborted because of its anatomical proximity to the motor strip. Postoperatively, fMRI was performed revealing motor activation at the pre and post central sulcus, which was used as a guide to a successful repeat intraoperative cortical stimulation and resection of the epileptic focus. Functional MRI is a useful tool for identifying motor or other eloquent cortices. During pre-operative planning of neurosurgical procedures involving cortical regions adjacent to eloquent cortex, the use of a multimodal approach can greatly improve accuracy and decrease the risk of post-operative deficits.
\end{abstract}

Manuscript accepted for publication March 2, 2011

${ }^{a}$ Department of Neurosurgery, Hackensack University Medical Center, New Jersey, USA

${ }^{b}$ Department of Radiology, OSF Saint Francis Medical Center, Peoria, IL, USA

${ }^{c}$ Department of Pediatrics and Neurosurgery, Hackensack University Medical Center, NJ, USA

${ }^{\mathrm{d}}$ Department of Radiology, Hackensack University Medical Center, NJ, USA

${ }^{\mathrm{e}}$ Northeast Regional Epilepsy Group, Comprehensive Epilepsy Center, Hackensack University Medical Center, NJ, USA

${ }^{f}$ Corresponding author: Mostafa El Khashab, drmelkhashab@yahoo.com

doi:10.4021/jnr104e
Keywords: Epilepsy surgery; Eloquent cortex; Functional mapping; Electrical cortical stimulation; fMRI

\section{Introduction}

Precise localization of eloquent cortex is critical in planning the extent of cortical resection in various neurosurgical procedures, including vascular, tumor and epilepsy surgery. This is especially true in neocortical, non-lesional, epilepsy surgery, where identification of the epileptic focus can be challenging and cortical resection involves cortical areas that are otherwise normal and functional. Therefore, avoiding eloquent cortical areas is crucial in reducing the risk of neurological deficits associated with the surgery. Direct electrical cortical stimulation is the most commonly used method to help identify eloquent cortex, and can be performed pre-operatively, when subdural grid electrodes are placed for identification of the epileptic focus, or intraoperatively, and is sometimes paired with cortical somatosensory evoked potentials [1-5]. However, other techniques have recently been introduced, and offer complementary or alternative methods that improve the precision of functional mapping. Those include magnetoencephalography (MEG), positron emission tomography (PET), functional MRI and electrocorticographic signal processing, all of which revealed comparative accuracy to cortical stimulation $[4,6,7]$. In some cases multiple modalities are used to minimize the risk of post-operative deficits. In fact, neither direct cortical stimulation nor evoked potentials are infallible in identifying the motor cortex $[8,9]$. The exact reason cortical stimulation sometimes fails is not entirely clear, and is frequently multifactorial.

We report the case of a patient with neo-cortical, nonlesional, intractable epilepsy who underwent evaluation for epilepsy surgery. Initial pre-operative and intraoperative cortical stimulation failed to identify the motor cortex, but fMRI pointed to the right location of the motor areas, allowing a repeat successful cortical stimulation, demonstrating the importance of a multimodal approach in helping identify eloquent cortex in difficult cases. 


\section{Case Report}

The patient was a 28 -year-old right-handed man with intractable epilepsy. He sustained a closed head injury at age 11 and started having seizures approximately 6 months later. He was also diagnosed with Attention Deficit Disorder. His seizures were characterized by a brief period of staring, followed by secondary generalization, sometimes associated with head version to the left. His seizures were refractory to multiple anti-epileptic drugs (AED) and he experienced weekly seizures for many years, sometimes associated with falls resulting in significant injuries and limb fractures. Brain MRI revealed developmental variant of a choroidal fissure cyst in the left hippocampus resulting in mild deformity of the distal body of the hippocampus. His PET scan showed only subtle decrease in metabolic activity in the anterior medial aspect of the right temporal lobe compared to the left side.

He underwent evaluation for epilepsy surgery. His phase I video-EEG monitoring revealed bilateral independent fronto-temporal interictal discharges and poorly localized seizures, frequently associated with diffuse, mostly bifrontal onset without consistent lateralization or localization. He underwent a survey study with bilateral frontal and temporal subdural strip electrodes inserted via burr holes, which revealed predominant seizure onset in the right hemisphere, mostly the right posterior frontal region. He subsequently had a right fronto-temporal craniotomy, with placement of a large lateral fronto-parietal grid and several strips sampling frontal and temporal regions. Several typical seizures were recorded, most of which with secondary generalization, indicating seizure onset in the posterior frontal and anterior parietal regions. Once enough information was collected, the patient was restarted on all his anti-epileptic medications and bedside cortical stimulation was performed to localize the primary motor cortex. Cortical stimulation was performed twice on two different days, but failed to reveal the motor cortex, despite using high output currents. The only functional area identified was the frontal eye field. Therefore, the patient was taken to the operating room and had intraoperative cortical somatosensory evoked potentials which failed to elicit a reliable phase reversal response. Extensive intraoperative cortical stimulation was then performed and again failed to reveal the motor areas. The areas of seizure onset corresponded anatomically to the anterior parietal cortex, and it was deemed risky to perform surgical resection in the absence of precise mapping of the motor cortex. Several pictures of the brain were taken and specific anatomical landmarks were recorded to mark the area of seizure onset. The procedure was aborted without resection. The exact reason for the failure of cortical stimulation to identify the motor cortex was not clear, and the possibility of cortical reorganization was considered. About a month later, the patient underwent functional MRI using a simple motor paradigm of 2
$\mathrm{Hz}$ finger tapping, revealing areas of motor activation in the posterior frontal regions bilaterally, corresponding anatomically to the expected location of the motor cortex, refuting the explanation of possible functional reorganization of the motor cortex. About a month later, a second craniotomy was performed and the area of seizure onset was identified using the pictures and anatomical landmarks recorded during the previous surgery. After craniotomy, the patient was allowed to wake up to the point of being able to communicate and follow directions. Initially, cortical somatosensory evoked potentials were attempted and revealed a reliable phase reversal. This was followed by cortical stimulation, starting in the area of phase reversal. EMG electrodes were inserted in several upper extremity muscles and the EMG signal was monitored continuously to help identify subtle contractions triggered by the stimulation. Motor responses were elicited in the face and upper extremity, allowing precise mapping of the motor strip. The area of seizure onset was posterior to the motor cortex and cortical resection was then performed. The patient was tested repeatedly during and after the resection without any deterioration in his motor function. There was no postoperative neurological deficit. His seizure improved markedly after surgery, with marked reduction in seizure frequency and severity. He did not have any secondarily generalized seizures anymore and his post-operative seizures are characterized by brief periods of behavioral arrest and staring, with rapid termination and return to baseline mental status.

\section{Discussion}

This case underlines the importance of using a multimodal approach to help identify eloquent cortex in difficult cases. Although direct cortical simulation remains the "Gold Standard" for functional mapping, functional MRI offers many advantages, including the non-invasive nature of the procedures, the ability to repeat it if necessary, and the use of a different physiological basis from cortical stimulation. The reason why initial cortical stimulation failed to trigger any motor response is not clear. The failure could not have been due to technical factors, since stimulation of the frontal eye field elicited reproducible eye deviation and head version to the left. Reorganization of motor cortex to other ipsilateral or even, contralateral cortical areas is a possibility, but was clearly not the case in this patient, since both functional MRI and repeated cortical stimulation revealed the motor cortex in the same region that was stimulated initially. We believe that the initial failure was due to changes in cortical excitability, possibly due to the effect of anti-epileptic medications and frequent seizures. After implantation of the subdural grid and strips, the patient was gradually tapered off his AEDs to record seizures. Several complex partial seizures with secondary generalization were recorded, and then the patient was 
reloaded with all his AEDs after being completely off medications for two weeks. Cortical stimulation was performed two days later. Seizures are known to cause changes in cortical excitability, and in this patient, it is possible that decreased cortical excitability was more pronounced because of the proximity of his seizure focus to the motor cortex and because of the frequent secondary generalization. Another important factor is the reintroduction of anti-epileptic drugs. In fact, several AEDs have been shown to lower the motor threshold and decreased cortical excitability measured by transcranial magnetic stimulation [10]. Reintroducing high doses of AEDs after a period of drug holiday may lead to cortical inhibition that will subside overtime with return of cortical excitability to a more stable baseline.

Direct cortical electrical stimulation is in general very reliable in identifying cortical areas preoperatively and intraoperatively. However, the technique can only be performed after craniotomy and usually cannot be repeated easily. Furthermore, as demonstrated in our case, there is a small chance of failure. Therefore, there is considerable effort to develop more non-invasive and reliable techniques for mapping functional areas [11], including fMRI, MEG [12] and PET [7], which have been demonstrated to be useful and highly accurate. Magnetoencephalography and PET can be very helpful, but demand special procedures and expensive equipment, not available in most institutions [12]. On the other hand, functional MRI can be acquired together with structural MRI, which is a routine presurgical procedure. Using fMRI guidance can improve the quality of the functional targeting whenever data are ambiguous by choosing the electrophysiological target that matches the activated area on fMRI [13]. Functional MRI offers many advantages. It is non-invasive and can be safely repeated if necessary. In addition, the mechanism of activation relies on changes in blood flow to specific cortical region, which is believed to correlate with electrophysiological activation. Furthermore, in patients with intracranial lesions that either deform cortical anatomy or potentially lead to displacement and reorganization of functional areas, cortical stimulation can be especially problematic and the use of functional MRI can be extremely helpful in identifying functional regions and help guide subsequent cortical stimulation if necessary.

Advances in anesthetic, radiologic and neurophysiologic techniques have greatly improved the safety of neurosurgical procedures and helped decrease morbidity and the risk of post-operative neurological deficits. Using a multimodal approach in identifying functional cortical areas can be very helpful to further reduce post-surgical morbidity.

\section{Abbreviations}

AED: Antiepileptic drug, EEG: Electroencephalography, EMG: Electromyography, fMRI: Functional magnetic reso- nance imaging, MEG: Magnetoencephalography, PET: Positron emission tomography.

\section{References}

1. Black P, Jaaskelainen J, Chabrerie A, Golby A, Gugino L. Minimalist approach: functional mapping. Clin Neurosurg 2002;49:90-102.

2. Duffau H, Capelle L, Denvil D, Sichez N, Gatignol P, Taillandier L, Lopes M, et al. Usefulness of intraoperative electrical subcortical mapping during surgery for low-grade gliomas located within eloquent brain regions: functional results in a consecutive series of 103 patients. J Neurosurg 2003;98(4):764-778.

3. Keles GE. Intracranial neuronavigation with intraoperative magnetic resonance imaging. Curr Opin Neurol 2004;17(4):497-500.

4. Roux FE, Boulanouar K, Ranjeva JP, Manelfe C, Tremoulet M, Sabatier J, Berry I. Cortical intraoperative stimulation in brain tumors as a tool to evaluate spatial data from motor functional MRI. Invest Radiol 1999;34(3):225-229.

5. Yingling CD, Ojemann S, Dodson B, Harrington MJ, Berger MS. Identification of motor pathways during tumor surgery facilitated by multichannel electromyographic recording. J Neurosurg 1999;91(6):922-927.

6. Fandino J, Kollias SS, Wieser HG, Valavanis A, Yonekawa Y. Intraoperative validation of functional magnetic resonance imaging and cortical reorganization patterns in patients with brain tumors involving the primary motor cortex. J Neurosurg 1999;91(2):238-250.

7. Krings T, Schreckenberger M, Rohde V, Spetzger U, Sabri O, Reinges MH, Hans FJ, et al. Functional MRI and 18F FDG-positron emission tomography for presurgical planning: comparison with electrical cortical stimulation. Acta Neurochir (Wien) 2002;144(9):889-899; discussion 899.

8. Horikoshi T, Omata T, Uchida M, Asari Y, Nukui H. Usefulness and pitfalls of intraoperative spinal motor evoked potential recording by direct cortical electrical stimulation. Acta Neurochir (Wien) 2000;142(3):257262.

9. Kombos T, Suess O, Funk T, Kern BC, Brock M. Intraoperative mapping of the motor cortex during surgery in and around the motor cortex. Acta Neurochir (Wien) 2000;142(3):263-268.

10. Joo EY, Kim SH, Seo DW, Hong SB. Zonisamide decreases cortical excitability in patients with idiopathic generalized epilepsy. Clin Neurophysiol 2008;119(6):1385-1392.

11. Larsen S, Kikinis R, Talos IF, Weinstein D, Wells W, Golby A. Quantitative comparison of functional MRI and direct electrocortical stimulation for functional 
mapping. Int J Med Robot 2007;3(3):262-270.

12. Stone SS, Rutka JT. Utility of neuronavigation and neuromonitoring in epilepsy surgery. Neurosurg Focus 2008;25(3):E17.

13. Pirotte B, Voordecker P, Neugroschl C, Baleriaux D,
Wikler D, Metens T, Denolin V, et al. Combination of functional magnetic resonance imaging-guided neuronavigation and intraoperative cortical brain mapping improves targeting of motor cortex stimulation in neuropathic pain. Neurosurgery 2008;62(6 Suppl 3):941-956. 